University of Nebraska - Lincoln

DigitalCommons@University of Nebraska - Lincoln

January 1989

\title{
Track “Core” Effects in Heavy lon Radiolysis
}

Robert Katz

University of Nebraska-Lincoln, rkatz2@unl.edu

Guo-Rong Huang

University of Nebraska-Lincoln

Follow this and additional works at: https://digitalcommons.unl.edu/physicskatz

Part of the Physics Commons

Katz, Robert and Huang, Guo-Rong, "Track "Core" Effects in Heavy lon Radiolysis" (1989). Robert Katz Publications. 75.

https://digitalcommons.unl.edu/physicskatz/75

This Article is brought to you for free and open access by the Research Papers in Physics and Astronomy at DigitalCommons@University of Nebraska - Lincoln. It has been accepted for inclusion in Robert Katz Publications by an authorized administrator of DigitalCommons@University of Nebraska - Lincoln. 
Published in Radiation Physics and Chemistry 33:4 (1989), pp. 345-349;

formerly, International Journal of Radiation Applications and Instrumentation. Part C. Radiation Physics and Chemistry

http://www.sciencedirect.com/science/journal/0969806X

Copyright (C) 1989 Pergamon Press plc. Used by permission.

Submitted March 15, 1988; revised June 24, 1988.

\title{
Track "Core" Effects in Heavy Ion Radiolysis
}

\author{
Robert Katz and Guo-Rong Huang \\ University of Nebraska-Lincoln, Lincoln, NE 68588-0111, U.S.A.
}

\begin{abstract}
By assuming that $\mathrm{HO}_{2}^{\bullet}$ radical production in water and $\mathrm{H}_{2}$ production in benzene are 2 hit processes, and applying the concepts of track physics, we are able to obtain a parametric fit to the yields of these reactions by heavy ion radiolysis from knowledge of the radial dose distribution about a heavy ion's path. We make no use of the concept of a track core, for no clearly definable track core appears in our calculations of the radial dose distribution. Instead we calculate an action cross section $\sigma$ from the assumed 2 hit response to $\gamma$-rays. The cross section is calculated from two fitted parameters, $E_{0}$, the $\gamma$-ray dose at which there is an average of 1 hit per target, and the target radius $a_{0}$. From the cross section, the target radius and the stopping power we calculate the $G$ value. While our model is not mechanistic, the assumed 2 hit process is consistent with hypotheses which have been offered as chemical models for these processes. Since a 2 hit process is more likely to take place in a high dose region, close to an ion's path, it may easily be attributed to a hypothetical track core in energy deposition, when indeed the response is a property of the detector.
\end{abstract}

\section{Introduction}

Three recent papers by La Verne et al. (LaVerne and Schuler, 1984, 1987; LaVerne et al., 1986) describe what are called track core effects in radiation chemistry, dealing with the production of $\mathrm{HO}_{2}^{\bullet}$ radicals in the heavy ion radiolysis of water and of $\mathrm{H}_{2}$ in the heavy ion radiolysis of benzene.

Since our calculations of the radial distribution of dose have shown that there is no "track core" (Waligorski et al., 1986) and that no track core is needed in the track structure analysis of the response of the Fricke dosimeter to energetic heavy ions (Katz et al., 1986), we have undertaken a track theory analysis (Katz, 1978) of these more recent data.

The response of the Fricke dosimeter differs in a significant way from these measurements in water and benzene, in that the $G$ values of the Fricke decline with an increase in LET, while these measurements display an increase in yield with an increase in LET. We take this as an indication that the Fricke dosimeter is a 1 hit process while these responses in water and benzene arise from 2 hit processes, and here describe the results of our attempt to ascribe parameters to these processes.

We note that our assignment of these as 2 hit processes is not inconsistent with the speculations of radiation chemists. Thus the production of $\mathrm{HO}_{2}^{\bullet}$ radicals is believed to require two water molecules, and to be produced in the second order reaction of hydroxyl radicals with hydrogen peroxide. Alternatively, a similar high order reaction involving the ionization of $\mathrm{H}_{2} \mathrm{O}_{2}$ has been proposed. The production of $\mathrm{H}_{2}$ from benzene may involve the bimolecular reaction of excited near neighbor molecules. There is presently no quantitative model of these processes, either mechanistic or parametric. This paper provides a parametric model which yields a re- markable fit to the heavy ion data. But we must point out that our parametric model is not simply a spline fit to the data, but is based on a track theory which is well developed for a wide range of detectors.

\section{Procedure}

Our procedure in track theory is to assume a functional form for the response of a system to $\gamma$-rays, given as the probability for activating the target of a detector as a function of the dose of $\gamma$-rays. These typically take the form of the multitarget or the multihit models of biological target theory. In the present case we have chosen the two or more hit function arising from the cumulative Poisson distribution, which gives the probability that a target will receive two or more hits as a function of the number of trials, $A$.

$$
P(2, A)=1-(1+A) e^{-A} .
$$

We take the number of trials at dose $E$ to be the ratio of $E$ to the dose $E_{0}$ at which there is an average of 1 hit per target so that the number of trials is

$$
A=E / E_{0} .
$$

This choice is in conflict with the existing data, for the authors have indicated that the yield is independent of dose of $\gamma$-rays. From the viewpoint of track physics one cannot obtain an increase in yield with an increase in LET if the yield from $\gamma$-rays is independent of dose. This point must be resolved experimentally in future, presumably with single pulses of energetic electrons of varying intensity.

Having chosen a functional form to describe the $\gamma$ ray dose-response relationship, we now make use of our calculations of the radial dose distribution about an ion's path to find the radial variation of activation prob- 
ability. To do this we first assign a size to the target region, which we take to be a chunky cylinder of radius $a_{0}$ whose axis is parallel to the ion's path. We then find the average dose within the target as a function of the radial distance of its axis from the ion's path. Given the dose in the target we find the activation probability, and integrate the activation probability radially to find the activation cross section, $\sigma$. Note that the activation cross section comes from all the excitations and ionizations about the ion's path, not either the "core" or the "penumbra" but both of these.

If $N$ is the number of targets per unit volume, the product $\sigma N$ is the number of activated targets per unit path length. Dividing by the stopping power $L$ yields the number of activated targets per unit energy deposited, which, properly translated into appropriate units is the $G$ value, the number of activated targets per $100 \mathrm{eV}$ of energy deposited by the ion. Thus

$$
G=\sigma N / L \text {. }
$$

Since the experiments of La Verne et al. were done with stopping particles we must integrate along the ion's path. We find the average value of the cross section by integrating $\sigma \mathrm{d} r$ over the range $R$ of the particle, and divide by $R$ to find the average cross section. To find the average stopping power we divide the initial energy $T$ by $R$. We take the number of targets per unit volume to be the reciprocal of the volume occupied by a sphere of radius $a_{0}$. We assign values of $E_{0}$ and $a_{0}$ which yield the best fits to the experimental data. Thus we strive to fit the experimental data with 3 parameters. The hittedness, $C=2$, the dose of $\gamma$-rays at which there is an average of 1 hit per target, $E_{0^{\prime}}$ and the radius of the target volume, $a_{0}$. Our model is statistical and parametric rather than mechanistic. No mechanistic explanation of these phenomena is presently available.

The calculated cross section yields the $G$ value only in the event that the activation is by a single passing ion. We refer to the damage produced by a single passing ion as "ion kill". At low fluence where ion paths are separated by a distance greater than the range of the most energetic $\delta$ ray, this is the only mode of activation. At high fluence the ion kill mode predominates for slow heavy ions. With fast heavy ions or with light ions, there is a significant residue of partially damaged targets which are not activated by a single ion. Indeed, the track of a single ion may resemble widely separated beads on a string. But targets between the activated ones may have been subactivated, as having experienced 1 hit when 2 are required. These may be activated by the $\delta$-rays from another passing ion at high fluence. We call this process " $r$ kill" for it resembles the manner in which targets are activated by the secondary electrons from $\gamma$ rays, and therefore follows the functional form of equation (1). Note that our model takes the probability for activation by a single electron and its secondaries to be negligible, for a 2 hit process. Hence we exclude the possibility of ion kill by electrons.

In the analysis of biological cell survival, the same argument is carried forward. We calculate as if the original cell population is exposed only to the ion kill mode of killing, which is described by an exponential function. We then assume that the survivors of the ion kill mode are the initial population for the $r$ kill mode, and that the fraction of the initial dose which is effective in the $r$ kill mode can be estimated from the fraction of surviving cells along an ion's track. This analysis enables us to parameterize cell survival in a heavy ion irradiation (Katz et al., 1971). The same argument has been used to interpret some results obtained in heavy ion lithography (Katz, 1983). To carry this type of analysis forward for the present case we would like to have information on the variation of yield with dose at a wide range of doses up to several times $E_{0}$.

In the present case we do not know the dose to which the materials, water and benzene, were exposed. If the fluence of ions is sufficiently low so that there is little possibility of overlap, only the ion kill contribution need be considered. At higher fluence, and for light ions like hydrogen or helium or lithium, and for fast heavy ions, we expect that the neglect of the $\gamma$ kill contribution may be significant, and our calculation based on ion kill alone should underestimate the experimental results.

\section{Results}

Our results are shown in Figures 1-3.

In Figure 1 we compare the experimental and calculated $G$ values for the production of $\mathrm{HO}_{2}^{\bullet}$ radicals in water with particles from $\mathrm{H}$ to $\mathrm{Ni}$ at initial energies up to $40 \mathrm{MeV}$. Our choice of parameters is based on the fit of our calculations to the more energetic ions in Figure 2. We must raise a question about the possibility of experimental problems with these measurements, for an alternative explanation, that our theory is in error for ions of energy below $40 \mathrm{MeV}$ is set aside by examination of Figure 3.

In Figure 2 we display the results for the same radical production with particles of energy up to $500 \mathrm{MeV}$. Here the fit of our model to data is good for $\mathrm{Ni}, \mathrm{O}$, and $\mathrm{C}$ ions, but there is disagreement for $\mathrm{Ne}$ ions, for which we have no explanation.

The parameters we have assigned to this process are $E_{0}=8.5 \times 10^{6} \mathrm{~Gy}, a_{0}=0.5 \mathrm{~nm}$, and the minimum number of hits required to activate a target $c=2$. These values imply that the "energy deposited in the target volume" when there is an average of 1 hit per target is about 28 $\mathrm{eV}$. The "radius" of the water molecule-approximated as a sphere and calculated from the density of water, the molecular weight, and Avogadro's number-is 0.2 nm.

Our results for the production of $\mathrm{H}_{2}$ in benzene are shown in Figure 3 . The parameters used are $E_{0}=8.5 \times$ 


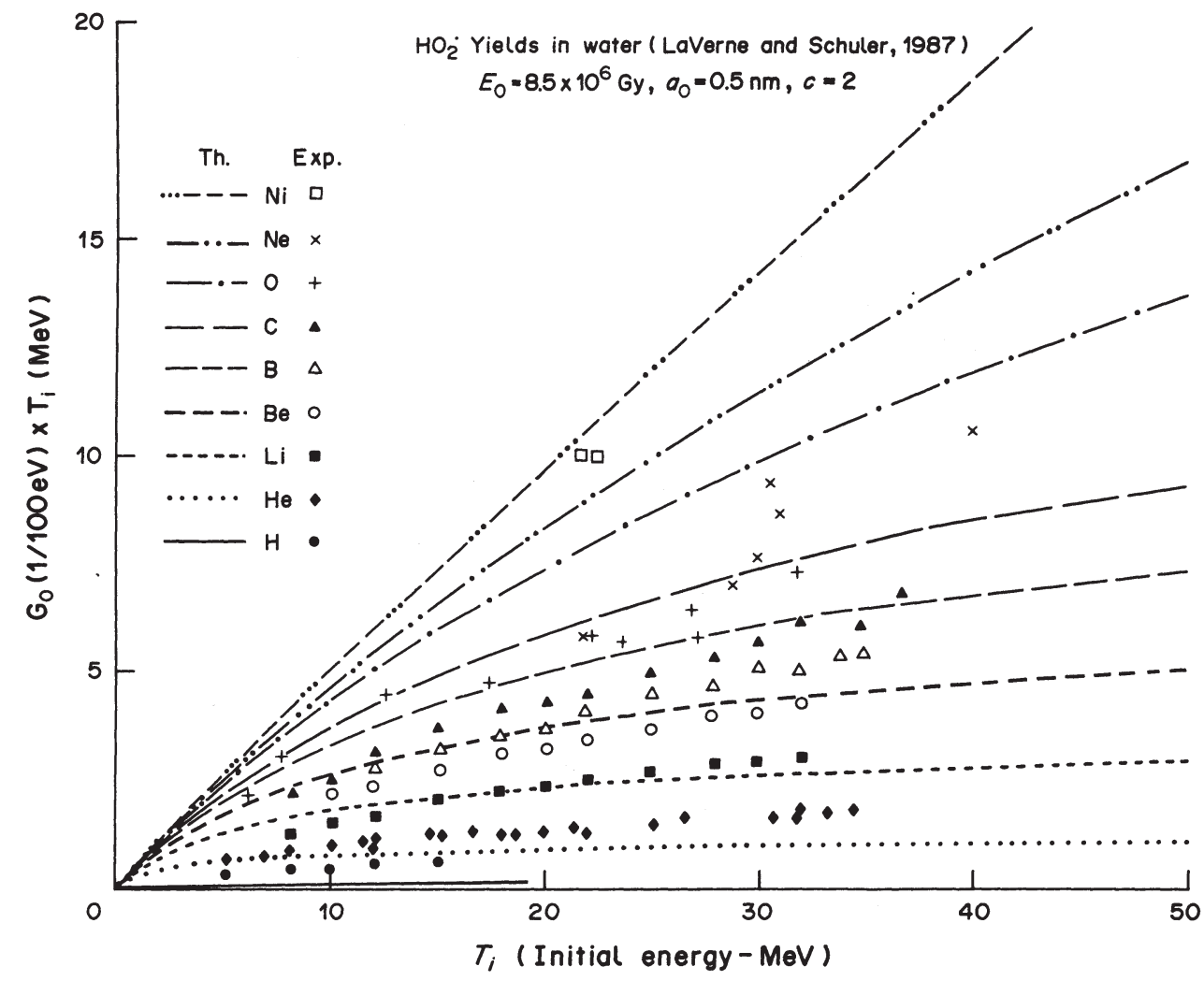

Figure 1. Experimental $G$ values multiplied by ion initial energies for $\mathrm{HO}_{2}^{\bullet}$ radical yields in water from heavy ions of initial energy up to $40 \mathrm{MeV}$ are shown as coded symbols. The curves are calculated from track theory using the parameters shown at the top of the figure. See text.

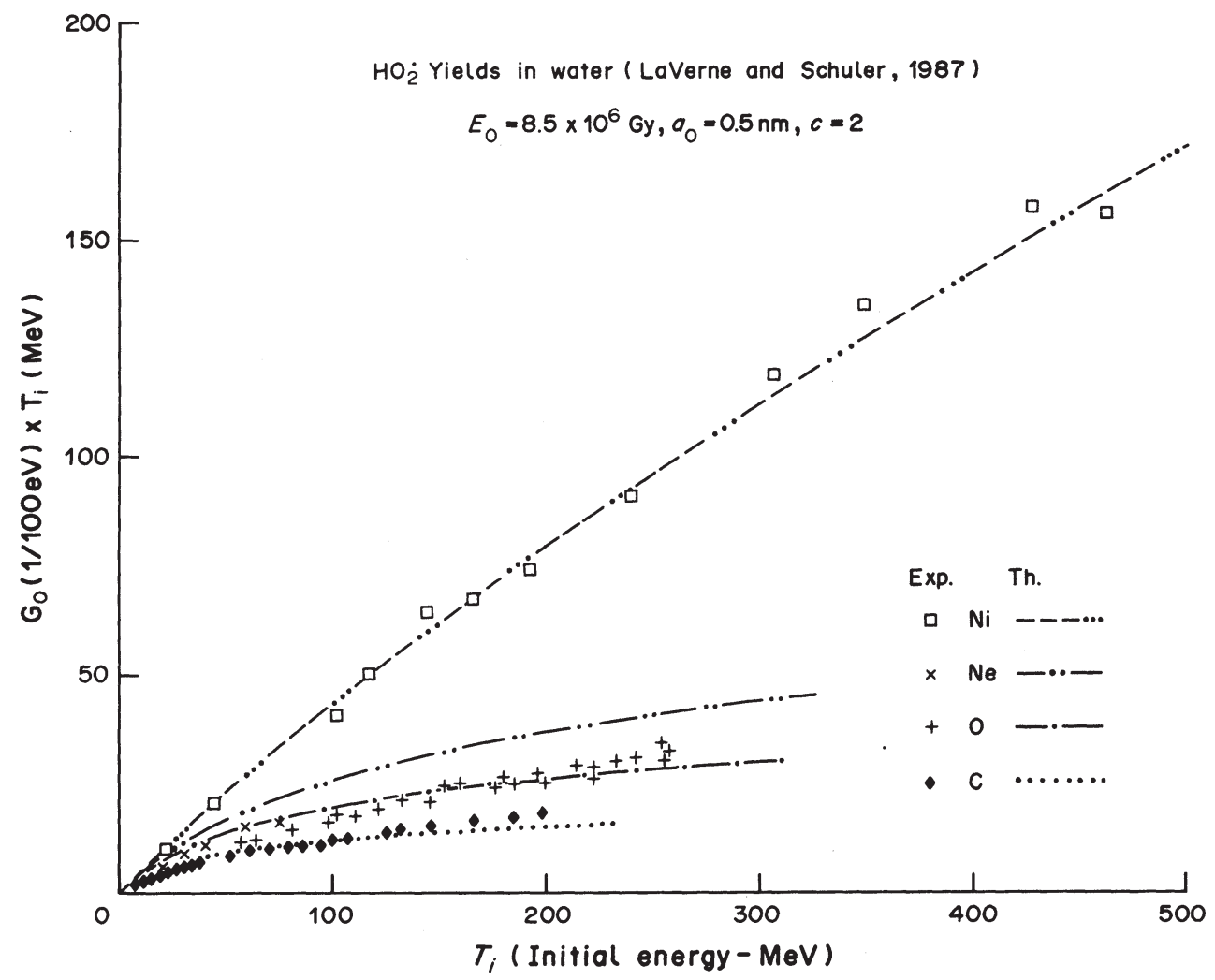

Figure 2. See caption for Figure 1. For ions of initial energy up to $500 \mathrm{MeV}$. 


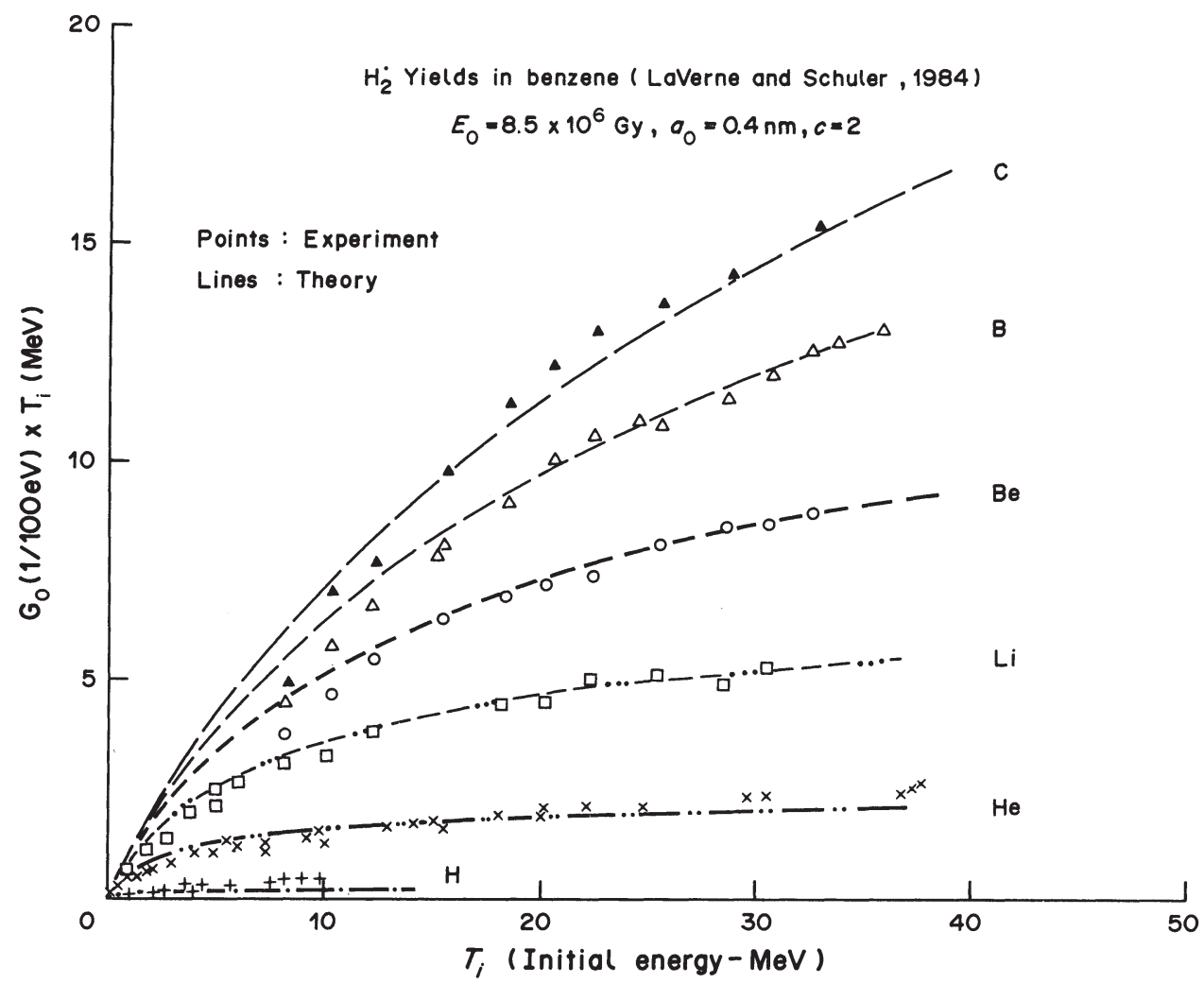

Figure 3. See caption for Figure 1. Data are for $\mathrm{H}_{2}$ yields in benzene.

$10^{6} \mathrm{~Gy}, a_{0}=0.4 \mathrm{~nm}$ and $c=2$. These values imply that the "energy deposited in the target volume" is about $14 \mathrm{eV}$ when there is an average of 1 hit per target. The "radius" of the benzene molecule-approximated as a sphere, and calculated from the density of benzene, the molecular weight, and Avogadro's number - is $0.33 \mathrm{~nm}$.

\section{Discussion}

If we insist that the number of targets per unit volume is the reciprocal of the volume per target, our parameters are tightly constrained, to about $5 \%$. We cannot further analyze the model for consistency with the data without knowledge of the doses and beam fluences used in these experiments. Our results for the heavy ions are compatible with the data only if the principal mode of activiation is by "ion kill," which is certainly the case if the fluence is small so that overlap of $\delta$-rays from adjacent ions is negligible, or if a large fraction of the sensitive targets intersected by the ions are activated.

It is inconsistent with our model that the $G$ values for $\mathrm{x}$-rays and light ions are independent of dose. Expansion of equation (1) in lowest order gives a quadratic response with dose for $\mathrm{x}$ - or $\gamma$-rays. The experimental findings claim the $G$ value for $x$-rays to be dose independent. This cannot be the case unless there are dose rate problems. To test our assertion one should irradiate these materials with pulses of energetic electrons whose duration approaches the transit time of a fast ion past a molecule, or at least is short compared to the lifetimes of excited states or intermediate radicals, or the time for the primary reaction products to diffuse away from their initial site by several molecular diameters.

The shape of our plots of yield vs. initial energy disagrees with the data for ions in Benzene of less than $15 \mathrm{MeV}$ of initial energy. For this we have no explanation. The problem may be with our use of an empirical expression for the effective charge for both stopping power and radial dose calculations for these relatively slow heavy ions. We have used calculations based on water to fit the benzene data.

We do not think it fortuitous that the fitted parameter $a_{0}$ is of the order of the molecular "radius" calculated from elementary considerations though we would not suggest that our procedures provide a precise determination of the molecular size.

Nevertheless, and in spite of all these caveats, it seems clear that one has no need for a specially contrived track core through which to analyze these data. The data will not make it possible to put a dimension onto the track core. Indeed we do not see how one can obtain more information by analyzing these data than is summarized in our parameters. The task of those who would attempt to build a mechanistic model of heavy ion radiolysis in 
these materials is now to explain the numerical values of our parameters.

These are clearly 2 hit processes. As a result most of the activation takes place near the ion's path. The core of which people speak will be different for each detector, for it reflects the properties of the detector as much as it reflects the radial dose distribution.

\section{Acknowledgement}

This work is supported by the U.S. Department of Energy.

\section{References}

Katz, R. (1978) Track structure theory in radiobiology and in radiation detection. Nucl. Track Detect. 2, 1.

Katz, R. (1983) Track structure interpretation of heavy ion lithography in relation to radiobiology and to the structure of etchable tracks in solid state nuclear track detectors. 8th Symp. on Microdosimetry, p. 135. Commission of the European Communities.
Katz, R., Ackerson, B., Homayoonfar, M., and Sharma, S. C. (1971) Inactivation of cells by heavy ion bombardment. Radial. Res. 47, 402.

Katz R., Sinclair, G. L., and Waligorski, M. P. R. (1986) The Fricke dosimeter as a 1 hit detector. Nucl. Tracks Radiat. Meas. 11, 301.

LaVerne, J. A., and Schuler, R. H. (1984) Track effects in radiation chemistry: core processes in heavy-particle tracks as manifest by the $\mathrm{H}_{2}$ yield in benzene radiolysis. J. Phys. Chem. 88, 1200.

LaVerne, J. A., and Schyuler, R. H. (1987) Production of $\mathrm{HO}_{2}$ in the radiolysis of water with heavy ions. In Third Workshop on Heavy Charged Particles in Biology and Medicine, p. B-3. Gesellschaft fur Schwerionenforschung $\mathrm{mbH}$, Darmstadt.

LaVerne J. A., Schuler, R. H., and Burns, W. G. (1986) Track effects in radiation chemistry: production of $\mathrm{HO}_{2}$ ' within the track core in the heavy particle radiolysis of water. J. Phys. Chem. 90, 3238.

Waligorski, M. P. R., Hamm, R. N., and Katz, R. (1986) The radial distribution of dose around the path of a heavy ion in Liquid Water. Nucl. Tracks Radiat. Meas. 11, 309. 